\title{
Management of metastatic castrate-resistant prostate cancer following renal transplantation
}

\author{
Tapper $\mathrm{A}^{1 *}$, Marin $\mathrm{M}^{2}$, Samarapungavan $\mathrm{D}^{2}$, Pam Jones $\mathrm{RN}^{3}$ and Hafron $\mathrm{J}^{1,3}$ \\ ${ }^{1}$ Department of Urology, Beaumont Health System, Royal Oak, Michigan, USA \\ ${ }^{2}$ Department of Nephrology, Beaumont Health System, Royal Oak, Michigan, USA \\ ${ }^{3}$ Michigan Institute of Urology, Troy Michigan, USA
}

\begin{abstract}
Prostate cancer $(\mathrm{CaP})$ is currently the most frequently diagnosed cancer in men in the United States. An increase in the incidence of cancer with viral etiologies is observed in transplant patients who undergo immunosuppression as part of transplant management. What is less well understood is the effect of immunosuppression on the development and progression of $\mathrm{CaP}$, particularly its effects on advanced disease. Additionally, there is little in the published literature regarding potential for drug-drug interactions between immunosuppressant medications and commonly used chemotherapeutic agents for advanced CaP. We found conflicting evidence regarding the effects of immunosuppression on prostate cancer development and progression, and particularly important for our case is the lack of evidence as to how it can alter progression of metastatic castrate resistant disease. Further, we found limited evidence of significant drug interactions between the currently used chemotherapy agents and immunosuppressant medications. As the survivorship of both transplant patients and patients with advanced CaP continue to improve, practitioners will have to become increasingly comfortable with the management of patients with both diseases.
\end{abstract}

\section{Introduction}

Prostate cancer $(\mathrm{CaP})$ is currently the most frequently diagnosed cancer in men in the United States [1]. It is well known that there is an increased incidence of cancers with viral etiologies in patients who are chronically immunosuppressed after renal transplants [2]. However, there are limited data in the literature regarding the incidence of $\mathrm{CaP}$ in this population and even less data on the incidence of metastatic castrate-resistant prostate cancer (mCRPC).

Recent therapeutic advances indicate a significant improvement of survival following the treatment of mCRPC. Treatment options include hormone therapy using an androgen biosynthesis inhibitor (abiraterone acetate) [3], androgen receptor antagonist (enzalutamide) [4], immunotherapy (Sipuleucel-T) [5], and alpha radiopharmaceutical therapy (radium 223) [6]. To our knowledge, none of them have been studied in the transplant patient population. In particular, there is little knowledge about the interactions of these agents with immunosuppressant medications. We present the case of a patient who developed mCRPC following renal transplantation. We examined the management of mCRPC in the transplant patient and assessed the potential effects of immunosuppression on the natural history of the disease as well as potential interactions between the immunosuppressant regimens and the novel agents.

\section{Case report}

An 82-year-old male with a past medical history of hypertension and resultant end-stage renal disease (ESRD) was diagnosed with $\mathrm{CaP}$ following an abnormal elevation of his serum PSA level. A transrectal ultrasound-guided prostate biopsy indicated adenocarcinoma of the prostate with Gleason $7(3+4)$ disease in 2 out of 12 prostate biopsy cores. He underwent an open radical retropubic prostatectomy in 1997, and a pathological examination revealed adenocarcinoma of the prostate T2aN0Mx with Gleason 6 disease and negative surgical margins. He developed a biochemical recurrence in 2010 and received salvage radiation therapy to the prostatic fossa. The patient's PSA level remained undetectable for 6 years following his salvage radiation therapy. He was initiated on hemodialysis in 2004 for end stage renal disease secondary to hypertension and subsequently underwent a cadaveric renal transplant on 4/21/2011. He received induction with Baciliximab followed by maintenance immunosuppression with Tacrolimus, Mycophenolate Mofitil, and Prednisone. Five years after the renal transplant, he developed another biochemical recurrence with PSA of $1.27 \mathrm{ng} / \mathrm{dL}$ and was started on continuous ADT. In 2016 he was diagnosed with mCRPC. Two successive rises in the PSA level were observed, peaking at $61.39 \mathrm{ng} / \mathrm{dL}$, and the castrate levels of testosterone were maintained by continuous ADT. Computed tomography (CT) imaging of his abdomen and pelvis indicated foci in enlarged lymph nodes within the left common iliac chain in the retroperitoneum (Figure 1), biopsy was discussed though never performed. Bone scintigraphy with Technetium-99m was negative for bone metastasis. The patient was started on Abiraterone Acetate therapy with weekly serum evaluation of his Tacrolimus levels, liver function, and renal function for the initial 8 weeks of therapy. After approximately 4 weeks of therapy, his Tacrolimus dosage was reduced (Figure 2). The patient exhibited an excellent response to Abiraterone Acetate, with the most recent PSA level being $3.24 \mathrm{ng} / \mathrm{dL}$. He has since been doing well with creatinine levels maintained well within normal range, last being 0.56 $\mathrm{mg} / \mathrm{dL}$, and urine output remaining appropriate without any voiding complaints. He continues to take immunosuppressant medications, Tacrolimus, Mycophenolic Acid, and Prednisone.

${ }^{*}$ Correspondence to: Alexander Tapper, Department of Urology, Beaumont Health System, Royal Oak, Michigan, USA, E-mail: Alexander.Tapper@beaumont.edu

Received: June 25, 2018; Accepted: July 12, 2018; Published: July 17, 2018 


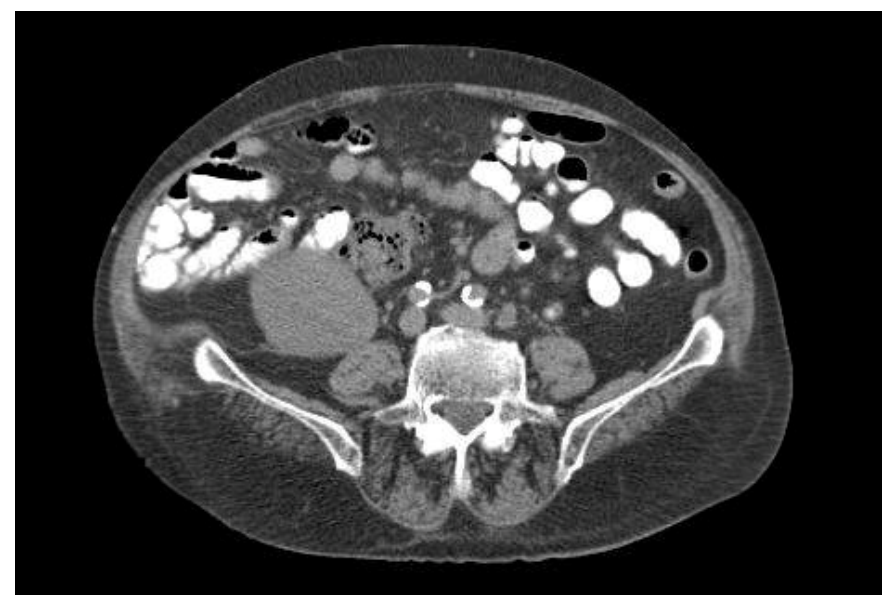

Figure 1. CT of the abdomen/pelvis without contrast.Arrows indicate the top of the renal graft coming into view (right) and the enlarged lymph nodes along the left common iliac chain (left)

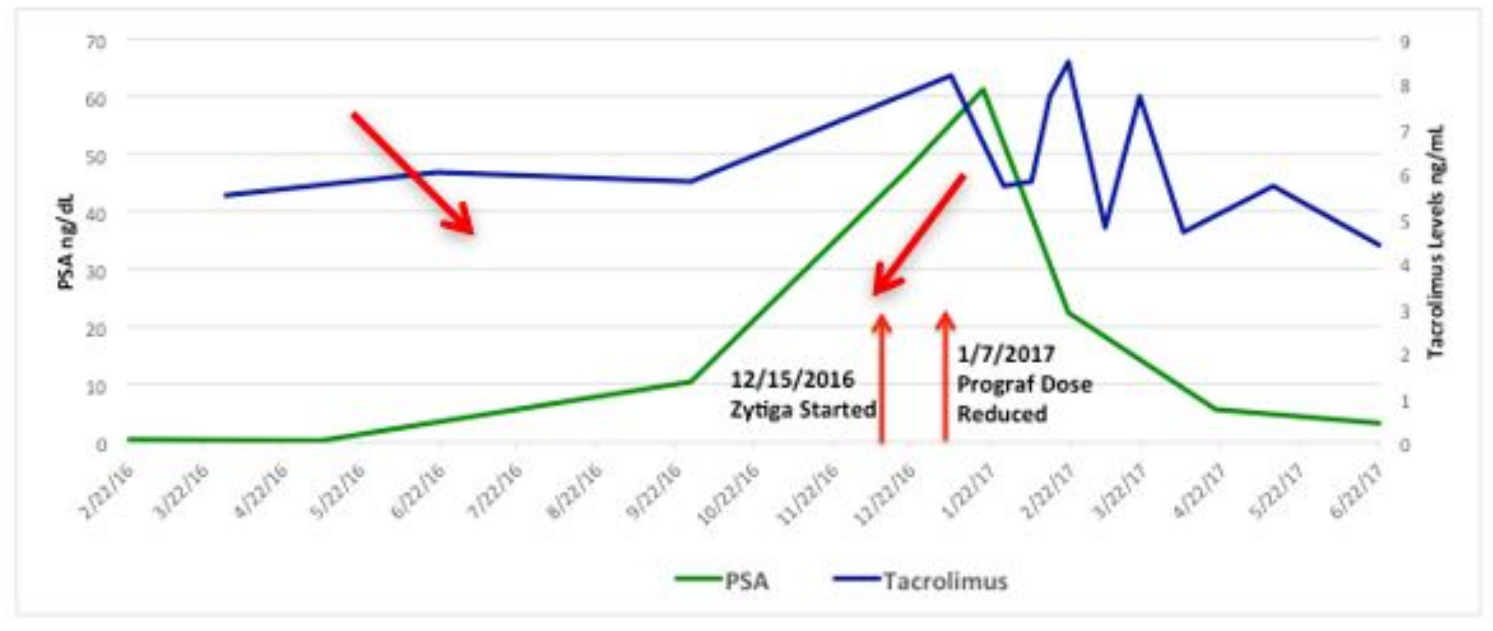

Figure 2. Graph demonstrating PSA levels and Tacrolimus values with the start date for Xytiga and the date of Prograf dose reduction labeled

\section{Discussion}

This complex case brings to light multiple clinical questions. As the transplant population continues to age and the modalities for increasing the overall survival continue to improve, it will become increasingly important to understand the diagnosis and management of $\mathrm{CaP}$ in these patients.

It is widely accepted that immune suppression after organ transplantation is associated with a marked increase in the rates of non-melanoma skin cancer, non-Hodgkin lymphoma, and Kaposi sarcoma. However, it is undetermined if other cancers occur at an increased frequency as well [2]. Moreover, is there a risk of the disease worsening with immune suppression following organ transplantation in patients with known malignancies that are not believed to be viral in etiology?

Multiple studies have demonstrated an increased risk of cancer development excluding non-melanoma skin cancer, as well as cancers associated with ESRD, and the overall risk of incident malignancies markedly increases following transplantation [2,7]. Hence, a significant increase in the incidence of $\mathrm{CaP}$ has yet to be demonstrated. Vajdic et al. demonstrated an increase in the risk of 25 specific cancers but did not find any increase in the risk of $\mathrm{CaP}$ in the post-transplant population [2].
Although large registry trials do not demonstrate an increased incidence of $\mathrm{CaP}$ in transplant patients, it has been shown that immunosuppression, including use of calcineurin inhibitors (CNIs) such as tacrolimus can contribute to the occurrence of post-transplant malignancies [8]. CNIs suppress the immune system by preventing interleukin-2 production. More pertinent to this case are the data supporting CNIs increasing the progression and aggressiveness of prostate adenocarcinoma tumor cells in both in vitro and in vivo models $[9,10]$. There have been suggestions that a judicious lowering of CNI dosing will lower the risk of cancer; however, to our knowledge, there has been no evidence that this will have a significant improvement on $\mathrm{CaP}$ management [11].

Furthermore, despite conflicting reports, there are limited data regarding the diagnosis and management of $\mathrm{CaP}$ in the transplant population. Binsaleh published a retrospective review of nine patients with renal transplants, all of whom were maintained on immunosuppressive therapy, and who were later diagnosed with $\mathrm{CaP}$. One of those patients was found to have poorly differentiated Gleason 8 disease, which was later characterized as a sarcomatoid variant. The patient developed spinal metastasis with subsequent development of allograft failure. Of the remaining eight patients, seven were noted to have stable PSA levels despite their immunosuppressed state [11]. Of note, these were denovo cases of $\mathrm{CaP}$ and therefore differ from our 
index patient in that his cancer preexisted the renal transplant and subsequent immunosuppression.

According to the NCCN Guidelines, Sipuleucel-T should be considered as the first-line therapy for the management of asymptomatic or minimally symptomatic mCRPC. However, the benefits of Sipuleucel-T would likely be mitigated by the activity of the immunosuppressant regimen. Tacrolimus acts by preventing dephosphorylation of a transcription factor responsible for the activation of T cells. Tacrolimus is a CNI; calcineurin is a calcium-dependent protein responsible for dephosphorylating NF-AT, a transcription factor involved in cytokine creation and activation. Mycophenolate is converted in the liver to an active metabolite that inhibits an enzyme responsible for purine synthesis and $\mathrm{T}$ and $\mathrm{B}$ cell proliferation [12]. To our knowledge, there are limited data regarding the use of Sipuleucel- $\mathrm{T}$ in the immunosuppressed population; however, the suppressive effects of medications such as Tacrolimus and Mycophenolate would likely mute the anti-tumor effects of Sipuleucel-T as it is a form of autologous immunotherapy mediated through $\mathrm{T}$ cell activation.

Abiraterone Acetate is an androgen biosynthesis inhibitor that has been demonstrated to improve overall survival both in patients with mCRPC who have received prior chemotherapy and in those who have not [3]. It is an oral chemotherapeutic agent that is metabolized by the cytochrome P450 group of enzymes. As a result, there is a significant risk of drug-drug interactions (DDIs) in patients taking these agents.

Specifically, Abiraterone Acetate is a substrate of sulfotransferase $2 \mathrm{~A} 1$ as well as CYP3A4. Additionally, it is a moderate inhibitor of CYP2D6 and a mild inhibitor of CYP2C8. This leads to potential interactions with a wide variety of medications, including $\beta$-blockers, serotonin reuptake inhibitors, anti-arrhythmics, opioids, rifampin, ketoconazole, and several others. A recent retrospective review examined the potential of DDIs in patients receiving Abiraterone for mCRPC. In 95 patients with $\mathrm{MCRPC}$, the median number of medications the patient took was 7 , with $80 \%$ of the cohort taking at least 5 medications. In this review, 49 patients were noted to be on medications found to have DDIs with abiraterone acetate, the majority being opioid pain medications. The second most common group of medications was cardiac antiarrhythmics. As one would expect, a history of chronic pain and poor ECOG performance status were associated with an increased incidence of DDIs with Abiraterone Acetate [13].

Both Tacrolimus and Mycophenolate are metabolized by the hepatic CYP450 enzyme system, and Tacrolimus relies heavily on the CYP3A4 enzyme system [14]. Tacrolimus is a medication that has a restricted therapeutic index and has demonstrated wide variability in blood levels and response following administration of a fixed dose [15]. Tacrolimus levels and liver function were monitored closely based on the unknown interaction between Abiraterone Acetate and the immunosuppressant our patient was receiving. He demonstrated no compromised renal function or change in liver function testing. His Tacrolimus level was adjusted in January 2017 to maintain the drug levels between 4 and $6 \mathrm{ng} / \mathrm{mL}$, as per Nephrology recommendations, it has since been maintained in the therapeutic range.

In addition to the interactions between the drugs themselves, the presence of underlying liver disease in patients with multiple comorbidities must be considered. Abiraterone Acetate has been noted to cause hepatotoxicity in $8 \%$ of patients in one study; however, this hepatotoxicity was a reversible elevation in transaminases and did not result in any deaths from hepatotoxicity-related events [3]. Nonetheless, this is worth considering prior to administering this agent to the patient, and a detailed discussion with a pharmacist is recommended to ensure that the patient is not on any other hepatotoxic medications. However, Abiraterone Acetate appears to be safe to use in immunosuppressed patients following a renal transplant because of the lack of DDIs with transplant medications as well as the lack of direct deleterious effects on renal function.

\section{Conclusion}

We presented the case of a patient with previously localized CaP treated with standard therapy who then became immunosuppressed following a renal transplant. The patient ultimately developed mCRPC. There are minimal data in the published literature regarding the management of these patients. This case is important because it suggests that Abiraterone Acetate is a safe and effective treatment option in transplant patients.

\section{Disclosures}

Dr. Hafron's COIs are as follows:

- Amgen Speaker

- Armune Bioscience Inc. Consultant and Speaker

- Dendreon Consultant and Speaker

- Jansen Speaker

\section{- United Physicians Board of Directors}

The remaining authors have no conflicts of interest and declare that there are no conflicts of interest regarding the publication of this paper.

\section{References}

1. Siegel R, Ma J, Zou Z, Jemal A (2014) Cancer statistics, 2014. CA Cancer J Clin 64: 9-29. [Crossref]

2. Vajdic CM, McDonald SP, McCredie MR, van Leeuwen MT, Stewart JH, et al. (2006) Cancer incidence before and after kidney transplantation. JAMA 296: 2823-2831. [Crossref]

3. Ryan CJ, Smith MR, de Bono JS, Molina A, Logothetis CJ, et al. (2013) Abiraterone in metastatic prostate cancer without previous chemotherapy. N Engl J Med 368: 138148. [Crossref]

4. Beer TM, Armstrong AJ, Rathkopf DE, Loriot Y, Sternberg CN, et al. (2014) Enzalutamide in metastatic prostate cancer before chemotherapy. $N$ Engl J Med 371: 424-433. [Crossref]

5. Kantoff PW, Higano CS, Shore ND, Berger ER, Small EJ, et al. (2010) Sipuleucel-T immunotherapy for castration-resistant prostate cancer. $N$ Engl J Med 363: 411-422. [Crossref]

6. Parker C, Nilsson S, Heinrich D, Helle SI, O'Sullivan JM, et al. (2013) Alpha emitter radium-223 and survival in metastatic prostate cancer. $N$ Engl J Med 369: 213-223. [Crossref]

7. Kasiske BL, Snyder JJ, Gilbertson DT, Wang C (2004) Cancer after kidney transplantation in the United States. Am J Transplant 4: 905-913. [Crossref]

8. Engels EA, Pfeiffer RM, Fraumeni JF Jr, Kasiske BL, Israni AK, et al. (2011) Spectrum of cancer risk among US solid organ transplant recipients. JAMA 306: 1891-1901. [Crossref]

9. François K (2008) Prostate cancer in renal transplant recipients. Nephrology Dialysis Transplantation 23: 2374-2380.

10. Pollard M (1997) Enhancement of metastasis of prostate adenocarcinoma cells by immune-suppressive cyclosporine A. Cancer Lett 111: 221-224. [Crossref]

11. Binsaleh S (2012) Diagnosis and treatment of prostate cancer in renal-transplant recipients. Int Urol Nephrol 44: 149-155. [Crossref] 
Tapper A (2018) Management of metastatic castrate-resistant prostate cancer following renal transplantation

12. Allison AC, Eugui EM (2000) Mycophenolate mofetil and its mechanisms of action. Immunopharmacology 47: 85-118. [Crossref]

13. Bonnet C, Boudou-Rouquette P, Azoulay-Rutman E, Huillard O, Golmard JL, et al. (2017) Potential drug-drug interactions with abiraterone in metastatic castrationresistant prostate cancer patients: a prevalence study in France. Cancer Chemother Pharmacol 79: 1051-1055. [Crossref]
14. van Gelder T (2002) Drug interactions with tacrolimus. Drug Saf 25: 707-712. [Crossref]

15. Provenzani A, Santeusanio A, Mathis E, Notarbartolo M, Labbozzetta M, et al. (2013) Pharmacogenetic considerations for optimizing tacrolimus dosing in liver and kidney transplant patients. World J Gastroenterol 19: 9156-9173. [Crossref]

Copyright: (C2018 Tapper A. This is an open-access article distributed under the terms of the Creative Commons Attribution License, which permits unrestricted use, distribution, and reproduction in any medium, provided the original author and source are credited. 